\title{
Iatrogenic circumflex coronary artery injury after mitral valve replacement
}

\author{
Luiza Bulak ${ }^{1}$, Piotr Brzozowski ${ }^{1}$, Krzysztof W. Reczuch², Wiktor Kuliczkowski $^{3}$ \\ ${ }^{1}$ Department of Heart Diseases, Jan Mikulicz-Radecki University Teaching Hospital, Wroclaw, Poland \\ 2Department of Heart Diseases, Wroclaw Medical University, Wroclaw, Poland \\ ${ }^{3}$ Department and Clinic of Cardiology, Wroclaw Medical University, Wroclaw, Poland
}

Adv Interv Cardiol 2021; 17, 3 (65): 315-316

DOI: https://doi.org/10.5114/aic.2021.109235

We present a case of a 57-year old man with a medical history of hypertension, coronary artery disease, heart failure, and hyperlipidemia. In June 2020 due to endocarditis the patient underwent valve surgery which required implantation of a mechanical valve (SJM 29). The intervention was complicated by postprocedural myocardial infarction (MI) expressed as ST segment elevation. The Heart Team convened ad hoc in the catheterization laboratory and decided on percutaneous coronary intervention $(\mathrm{PCl})$. Coronary angiography revealed total occlusion of the left circumflex artery $(C x)$ at the origin of the large first marginal obtuse artery (Mo), which additionally presented proximal stenosis (Figure $1 \mathrm{~A}$ ). The coronary angiography performed before the operation showed no signs of coronary atherosclerosis in these locations. The surgeon's description of the operation, together with the coronary angiogram, suggested that the $\mathrm{Cx}$ stenosis was associated with annuloplasty suture ligation, which is a rare, but possible complication due to proximity of the $C x$ and mitral annulus [1-3]. After guidewires crossed the Mo and Cx, predilatation with $2.5 \mathrm{~mm}$ and $3.0 \mathrm{~mm}$ non-compliant (NC) balloons was performed. After the balloons were pulled out, a control contrast injection was made. Cx narrowing appeared again, probably in the place where tissues or stitches caused pressure. Due to recurrent $\mathrm{Cx}$ stenosis after ballooning the final decision was made to implant a stent. Using the culotte technique and Guideliner system, two drug-eluting stents (DES) were placed: $2.5 \times 26 \mathrm{~mm}$ Resolute Onyx in the Cx and after proximal optimization (POT) $4.0 \times 18 \mathrm{~mm}$ Resolute Onyx in the large Mo. Finally "kissing balloons" inflation with NC $3.0 \mathrm{~mm}$ and $4.0 \mathrm{~mm}$ balloons was performed and re-POT with the NC $4.0 \mathrm{~mm}$ balloon. We obtained a good procedural effect, but still with some pinching in the stent in the area of Cx former amputation (Figure $1 \mathrm{~B}$ ). Transthoracic echocardiogram showed left ventricular hypokinesia and depressed left ventricular function (ejection fraction between 35\% and 40\%).

Three months later the patient started to complain about CCS II chest pain and because of presumed high restenosis risk, the patient was re-invited to our department in September 2020 for follow-up coronary angiography. An echocardiogram showed left ventricle ejection fraction (LVEF) of $42 \%$ and regular function of the mechanical valve.

The catheterization showed $90 \%$ restenosis in the DES which had been placed in the Cx exactly in the spot of the former amputation (Figure $1 \mathrm{C}$ ). After predilatation intravascular ultrasound (IVUS) was used and confirmed in-stent restenosis, after which the $3.0 \times 8 \mathrm{~mm}$ DES (Resolute Onyx) was chosen and with the $T$ and small protrusion (TAP) technique was placed. For proper stent expansion "kissing balloons" and POT methods with two $4.0 \times$ $6 \mathrm{~mm} \mathrm{NC}$ balloons were used (Figure $1 \mathrm{D}$ ). IVUS showed $8.4 \mathrm{~mm}$ minimal stent area (MSA) in double layer stents area in the $\mathrm{Cx}$ and proved a good procedural effect.

The patient was contacted in April 2021 and since the last $\mathrm{PCl}$ he has been doing fine without any chest pain.

It should be noted that mitral valve surgery can injure the $C x$, because of the short distance between the $C x$ and the mitral annulus [1-3]. This case highlights the importance of close monitoring of a patient after mitral valve surgery. According to the literature, this kind of iatrogenic injury can cause different clinical symptoms: ST-segment changes but sometimes only ventricular arrhythmias or wall motion abnormalities without overt ECG changes [4]. Treatment options include surgery with repeated mitral operation with or without $C x$ by-passing

Corresponding author:

Luiza Bulak, Department of Heart Diseases, Jan Mikulicz-Radecki University Teaching Hospital, Wroclaw, Poland, e-mail: luizabulak@op.pl Received: 16.05.2021, accepted: 16.08.2021. 

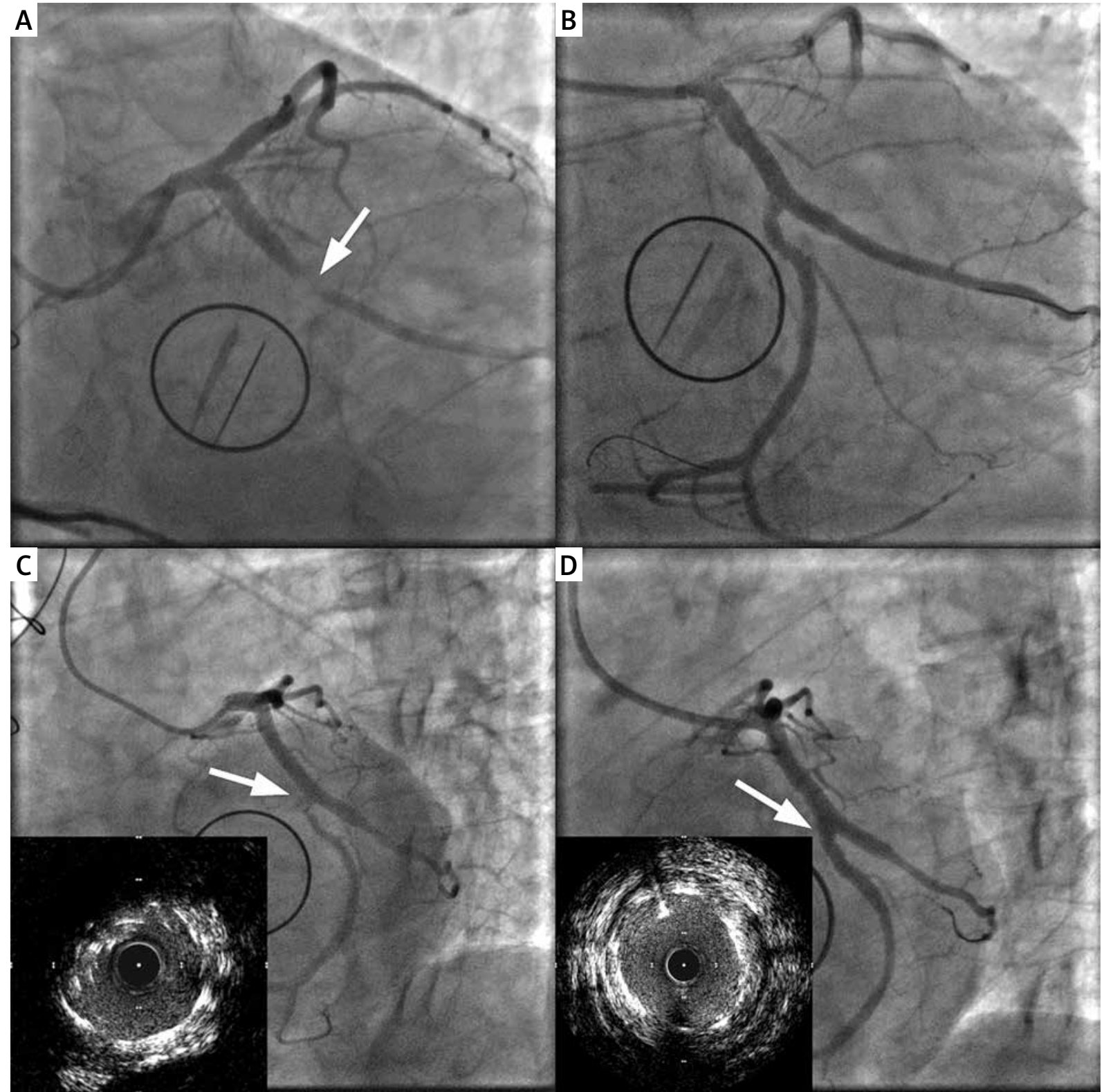

Figure 1. Angiography. A - Angiogram view (LAO/CAUD): white arrow shows occlusion of the left circumflex artery (June 2020). B - Post-stenting angiography (June 2020). C - Control coronarography after 4 months: arrow shows in-stent restenosis + IVUS view (September 2020). D - Final result after additional stenting + final IVUS (September 2020)

or percutaneous intervention [4]. In our case the unexpected Cx injury, which led to postprocedural $\mathrm{MI}$ and recurrent narrowing after ballooning during $\mathrm{PCl}$, forced us to implant a stent. Despite the good immediate result, fast restenosis occurred with the need for repeat $\mathrm{PCI}$ with implantation of a bigger stent. Presumably in such cases implantation of a DES with thicker struts and/or higher radial force would be a way to obtain better results without high restenosis risk.

\section{Conflict of interest}

The authors declare no conflict of interest.

\section{References}

1. Fábián B, Osadczuk A, Bárány L, et al. Real 3D visualization of the circumflex artery surrounding the mitral annulus. Thorac Cardiovasc Surg 2020, Oct 21 [Epub ahead of print]; doi: 10.1055/s-0040-1715181.

2. Kaklikkaya I, Yeginoglu G. Damage to coronary arteries during mitral valve surgery. Heart Surg Forum 2003; 6E128-42.

3. Hage A, Hage F, Sridhar K, et al. A novel hybrid approach to iatrogenic circumflex artery injury after mitral repair. Semin Thorac Cardiovasc Surg 2020; 32: 486-9.

4. Virmani R, Chun PK, Colonel L, et al. Suture obliteration of the circumflex coronary artery in three patients undergoing mitral valve operation. J Thorac Cardiovasc Surg 1982; 84: 773-8. 